\title{
Brachial Mycotic Pseudoaneurysms Due to Prosthetic Valve Infective Endocarditis: A Case Report and Review of the Literature
}

\author{
Ovidiu Stiru, MD, PhD, Roxana Carmen Geana, MD, Liana Valeanu, MD, Tulin Adrian, MD, PhD, \\ Laura Raducu, MD, PhD, Vlad Anton Iliescu, MD, PhD
}

Department of Cardiovascular Surgery, Faculty of Medicine, Carol Davila University of Medicine and Pharmacy, Bucharest, Romania; Prof. Dr. C. C. Iliescu Emergency Institute for Cardiovascular Diseases, Bucharest, Romania

\section{ABSTRACT}

Brachial mycotic pseudoaneurysms (BMPA) are a rare complication of infective endocarditis (IE), but potentially could be a limb-threatening condition. We present the case of a 38-year-old male referred to our department, complaining of the sudden onset of a painful pulsatile mass $5 \mathrm{x}$ $10 \mathrm{~cm}$ in the right antecubital fossa that slowly progressed over time. Two years before this, he underwent aortic and mitral valve replacement with mechanical prosthetic valves and tricuspid annuloplasty for IE with methicillin-susceptible Staphylococcus aureus after a six-week course of intravenous antibiotherapy with oxacillin. Clinical examination of the right upper limb revealed a pulsatile and compressible mass with a normal temperature and without other clinical signs of inflammation. Pulse of the axillary artery, brachial and radial arteries were palpable. He was diagnosed by Doppler ultrasonography and digital subtraction angiography with BMPA.

Furthermore, transesophageal echocardiography (TEE) revealed normal function of the aortic and mitral prosthetic valve with no signs of prosthetic valve endocarditis and no feature of congestive heart failure. Considering these clinical findings, surgical treatment was planned. He underwent re-section of the brachial pseudoaneurysm and arterial reconstruction. One year after the pseudoaneurysm resection, evolution was excellent. This manuscript presents this rare, uncommon complication after IE and also reviews the available surgical management strategies for this pathology.

\section{INTRODUCTION}

Infectious invasion of the native arterial wall in the absence of any trauma, surgical, or endovascu-lar arterial approach results in pseudoaneurysm formation. Osler defined these as mycotic aneu-rysms [Osler 1885]. Pseudoaneurysms are pulsatile hematomas, due to disruption of the arterial wall with a persistent communication between the arterial vessel and

Received March 7, 2020; received in revised form April 23, 2020; accepted April 23, 2020.

Correspondence: Ovidiu Stiru MD, PhD, Prof. Dr. C. C. Iliescu Emergency Institute for Cardiovascular Diseases Sos. Fundeni 258, sector 2, 022328 Bucharest, Romania; phone/fax: +400722207286 (e-mail: ovidiu_stiru@yahoo.com). a localized hematoma. The most commonly affected arteries are cerebral arteries, followed by the aorta. Brachial arteries are affected in about 3-10\% of cases [Stengel 1923; Brown 1984; Patra 2001; Cakalagaoglu 1999]. Ruptured peripheral mycotic pseudoaneurysms lead to a high prevalence of limb loss and even mor-tality rate [Stengel 1923]. The most common causes of mycotic peripheral pseudoaneurysms are septic emboli arising from native cardiac valves endocarditis, intravenous drug abuse, invasive catheterization procedures, and prosthetic valve endocarditis. The upper limb is affected in $10 \%$ of cases of mycotic pseudoaneurysms [Leon 2008], and the prevalence is estimated to be about $0.03 \%$ per year [Tsao 2002].

\section{CASE PRESENTATION}

The present study analyzed the clinical data of a 38-yearold male, who was admitted with complaints of a painful $5 \mathrm{x} 10 \mathrm{~cm}$ pulsatile mass in the right antecubital fossa. His medical history included IE with methicillin-susceptible Staphylococcus aureus two years before, for which he underwent aortic and mitral valve replacement with mechanical prosthetic valves and tricuspid annuloplasty. A one-week course of oxacillin antibiotherapy was administered before negative blood cultures were obtained and then continued for another six weeks before surgery. The intraoperative bacterial cultures of the valves were negative, and the postoperative course was uneventful. One year after surgery, no functional anomalies or signs of IE were observed for the prosthetic aortic and mitral valves. There was no following history of trauma, instrumentation (venous puncture, arteriography, dialysis, drug abuse), or any intervention or surgery in the affected area. Two years after cardiac surgery, the patient complained of pain and difficulty with movement of his right arm. The appearance of a painful pulsatile mass that progressively had grown was noted. Right arm examination showed an ovoid swelling $5 \times 10$ centimeters on the medial aspect with visible pulsation, at 10 centimeters above the medial epicondyle. The color of the skin overlying the swelling mass was the same as that of the surrounding skin. Palpation revealed a non-tender, pulsatile, compressible, non-fluctuant mass with the same temperature as that of the surrounding skin, which was non-reducible, not blanching on pressure, and not attached to the overlying skin or 


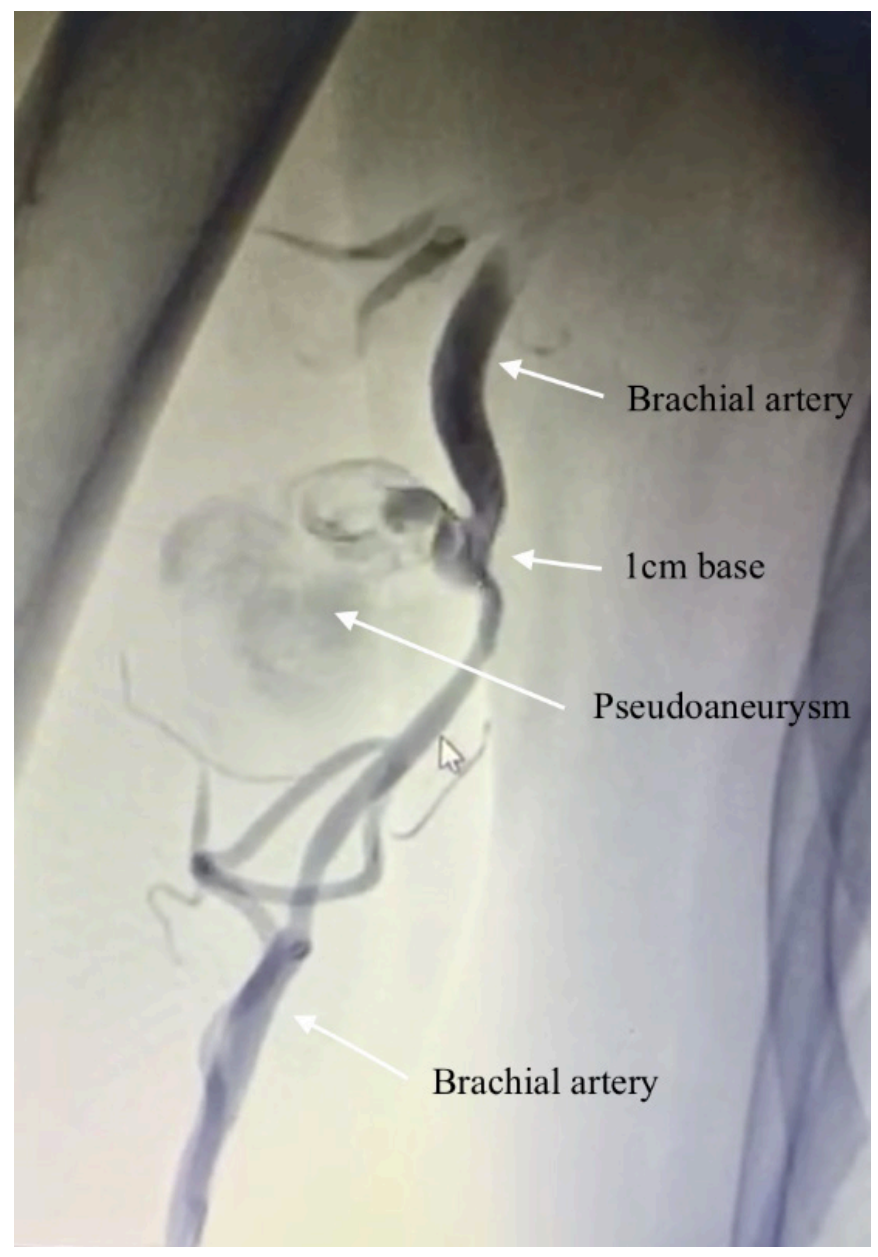

Figure 1. Angiography view showing a brachial mycotic pseudoaneurysm (BMPA) with a $1 \mathrm{~cm}$ base in the third distal of the upper arm.

underlying muscle or bone, with no skin necrosis or ulceration. The right arm was found to be warm with good capillary filling. There were no symptoms or signs of peripheral vascular ischemia secondary to aneurysm thrombosis or rupture and no neurologic symptoms nor signs due to direct compression. The radial and ulnar arteries had a palpable distal pulse. The remainder of the systemic examination was unremarkable, and normal white blood cell (WBC) count and hemoglobin level was noted. $\mathrm{C}$ reactive protein value was $64 \mathrm{mg} / \mathrm{L}$ and procalcitonin level was $0.2 \mathrm{ng} / \mathrm{mL}$. The bacterial screening, including blood cultures, was negative. Duplex ultrasonography of the right arm showed the presence of a pulsatile mass, measuring approximately $5 \mathrm{~cm}$ in diameter at $10 \mathrm{~cm}$ above the bifurcation of the brachial artery. The axillary, brachial, ulnar, and radial arteries were patent, with regular caliber throughout their courses, with normal triphasic flow. Angiographic examination revealed a pseudoaneurysm located in the middle of the brachial artery with a $1 \mathrm{~cm}$ broad base with permeable brachial, ulnar and radial arteries (Figure 1). TEE confirmed normal function of the prosthetic cardiac valves, no signs of prosthetic valve IE, and normal left and right ventricular function.

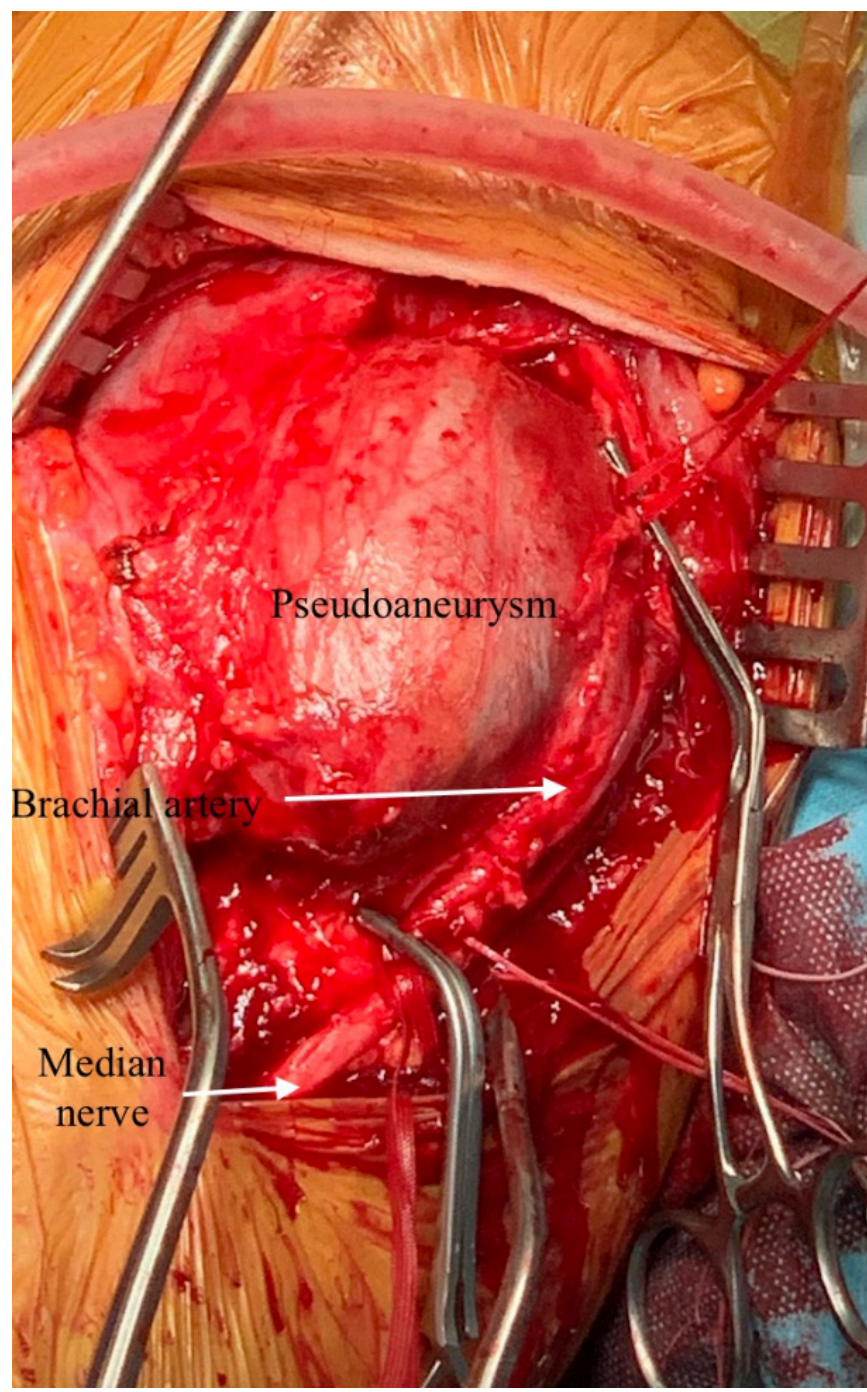

Figure 2. Intraoperator view-pseudoaneurysm of the brachial artery.

Treatment options were thoroughly discussed and explained to the patient. Due to pseudoaneurysm rupture risk, it was decided to immediately proceed with the surgical repair and also to initiate intravenous antibiotherapy with vancomycin and gentamycin. A right supraclavicular brachial plexus block was performed. The patient was placed in the supine position with an abducted right upper limb. A longitudinal incision was made on the medial side of the arm centered by the pseudoaneurysm and extended proximal and distal to expose the pseudoaneurysm and brachial artery (Figure 2). The pseudoaneurysm of the brachial artery was isolated from the median nerve, and it was excised after obtaining adequate proximal and distal brachial artery control. Extensive mobilization of both cut ends of the brachial artery was achieved, and brachial artery reconstruction was performed in an end-to-end fashion without the need of interposition or bypass grafting (Figure 3). The biopsy sample revealed pseudoaneurysm of the brachial artery with mural thrombus within. The microscopic appearance of the 


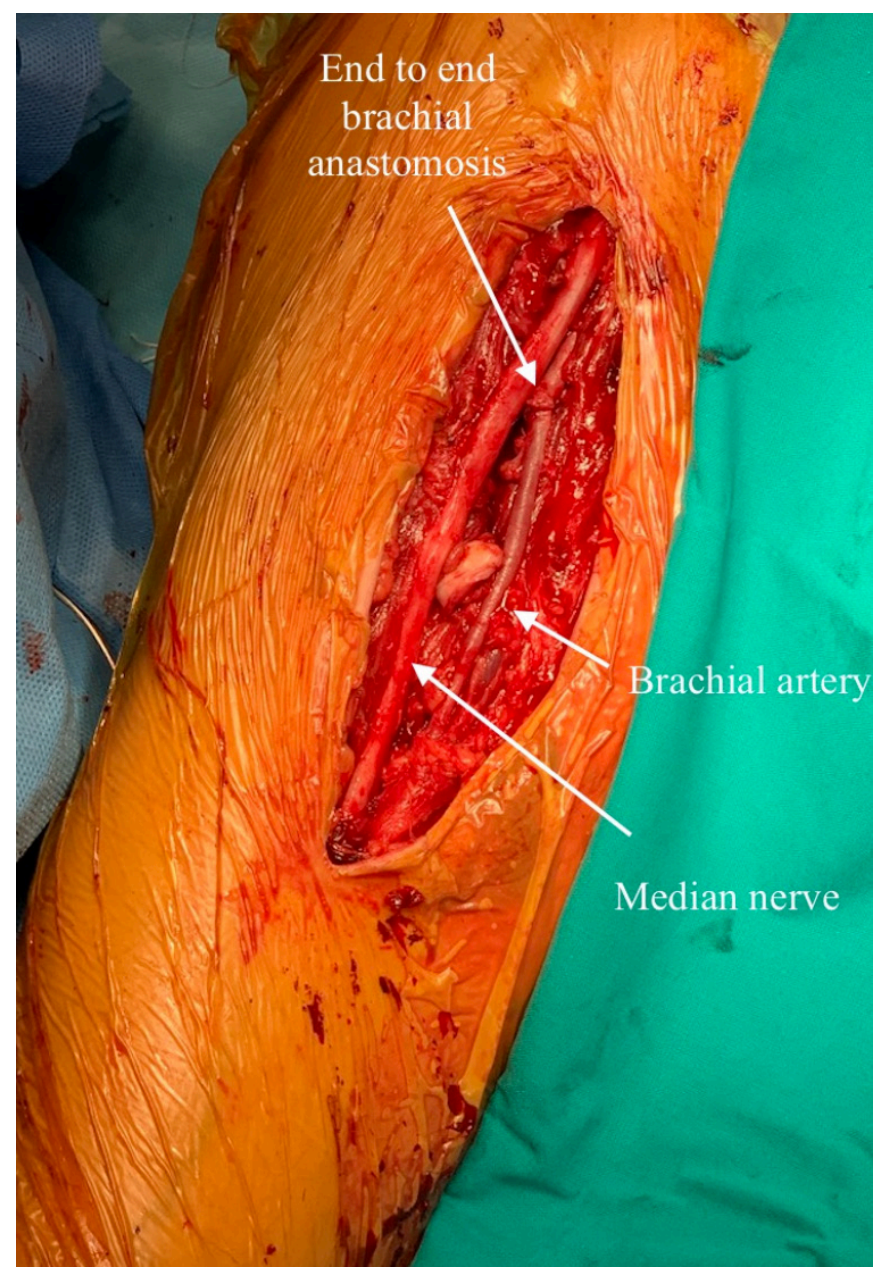

Figure 3. Intraoperator view-brachial artery reconstruction. Final aspect.

resected aneurysmal wall revealed structures of the arterial wall composed of medial and adventitial layers, suggestive of pseudoaneurysm of the brachial artery. Even though the intraoperative bacterial cultures were negative, and procalcitonin values reached the reference level one week after surgery, a postoperative antibiotic course was administered for six weeks. The vancomycin was switched to oxacillin after the negative result of the bacterial cultures, and the gentamycin was discontinued after two weeks. One year's scheduled control Duplex ultrasonography revealed normal flow in the brachial, radial, and ulnar arteries, and TEE showed normal prosthetic cardiac valves function.

\section{DISCUSSION}

BMPA sequela of infective endocarditis is uncommon. Mycotic peripheral pseudoaneurysms are rare but represent a severe complication of IE. Risk factors for brachial aneurysm include injuries (penetrating, blunt trauma), iatrogenic (orthopedic, arterial catheterization, hemodialysis fistula, failure of anastomosis after arterial reconstruction or repair, arterial graft degeneration), IE, con-genital arterial defect, osteochondroma, neurofibromatosis, inflammation (vasculitis, pancreatitis), hemophilia, collagen disorders (Marfan and Ehlers-Danlos syndrome), and erosion from malignancy or intravenous drug abuse. The brachial artery is the most frequently reported site involved in upper extremity mycotic aneurysms [Patra 2001]. The mechanism incriminated in the occurrence of BMPA is septic embolization of vegetation arising from IE, which lodges within the arterial vasa vasorum wall, with subsequent spread of infection throughout the vessel wall [Leon 2008; Mylonakis 2001]. It is assumed that prosthetic valve endocarditis is the most common causative factor, followed by intravenous drug use. Peripheral angiography can be used to confirm the diagnosis. Treatment delay causes upper limb ischemia, venous distal limb edema, local cutaneous erosion and hemorrhage, and adjacent neurological compression. The accepted surgical principles include resection of the affected brachial segment, pseudoaneurysm excision, and debridement of all necrotic and infected tissue; it is followed by immediate revascularization either by simple or complex repair. Patients can present with no distal vascular symptoms or ischemia due to thromboembolic complications. For pseudoaneurysms with a small narrow pedicle, with no distal embolic complication, simple repairs with interrupted or running 6.0 polypropylene sutures, or a venous patch angioplasty is preferable. When the resected brachial artery segment is short, it is sometimes possible to mobilize both edges of the brachial artery and perform an end-toend arterial anastomosis. Caution should be exercised to avoid undue tension because this may lead to suture line disruption or in situ thrombosis. In a more complex repair for pseudoaneurysms with a large, broad base, extensive arterial segment resection is necessary, and the repair must be done by bypass interposition with autologous venous graft or synthetic conduit. In patients presented with limb ischemia due to thromboembolic complications, brachial, radial, and ulnar artery Fogarty embolectomy may be necessary. Some of these procedures may result in serious complications such as upper limb amputation, brachial plexus involvement, and flexion contractures. The brachial artery affected can be excised and ligated as definitive surgical therapy if adequate collateral vessels exist. When rapidly performed, surgical treatment was associated with favorable outcomes. While the surgery was the gold-standard treatment, several less invasive alternative approaches include ultrasoundguided percutaneous thrombin injection, ultrasound-guided compression obliteration, and endovascular procedures, which include exclusion of pseudoaneurysm. Thrombin percutaneously can be injected directly into a pseudoaneurysm, causing it to clot. The technique is minimally invasive and has become the procedure of choice; it is relatively safe with a success rate ranging from $75 \%$ to $89 \%$ [Gürel 2012] Endovascular procedures also have been reported, with a covered-stent graft that may be placed endovascularly across this hole to exclude it [Stiru 2018], but its role in infectious pseudoaneurysms has yet to be defined [Nishimura 2015]. This technique has a high success rate with-out the need for open surgery. A significant complication in mycotic pseudoaneurysms is the infection of the stent, which determines the limited value of this procedure in EI. However, stent migra-tion and fracture also are possible and 
require regular monitoring. As with all mycotic pseudoaneurysms, it is essential to treat the source of the infectious emboli with intravenous antibiotics and valvular replacement in the case of native valve IE. Endovascular or surgical repair always should be considered at the time of diagnosis, even when BMPA is asymptomatic. Postoperatively, antibiotics should be given for at least six weeks to three months [Gonzalez 2014].

In our case, the development of BMPA did not coincide with EI. Thus, our treatment plan consisted of surgical resection of the pseudoaneurysm and postoperative antibiotic therapy as prophylaxis of prosthetic valve endocarditis. However, if BMPA is diagnosed simultaneously with IE on native or prosthetic valves, we consider that surgical treatment of the pseudoaneurysm should be performed prior to treatment of endocarditis.

The multidisciplinary heart team consisting of the infectious disease doctor, cardiologist, and cardiac surgeon should decide the timing of the cardiac surgery, depending on the patient's hemodynamic and infectious status.

\section{CONCLUSION}

Surgical repair always should be considered at the time of diagnosis. It has been associated with favorable outcomes. Complete excision of the affected artery and total removal of the pseudoaneu-rysm and surrounding tissues with preservation of the nerve, vein, and brachial artery revascularization should be considered the standard of care. It is essential to treat the source of the infectious emboli with intravenous antibiotics for at least six weeks up to three months.

\section{REFERENCES}

Brown SI, Busuttil RW, Baker JD, Machleder HI, et al. 1984. Bacteriologic and surgical determi-nants of survival in patients with mycotic aneurysms. J Vasc Surg 1:541e547.

Cakalagaoglu C, Keser N, Alhan C. 1999. Brucella-mediated prosthetic valve endocarditis with brachial artery mycotic aneurysm. J Heart Valve Dis 8:586-590.

Gonzalez I, Sarria C, Lopez J, Vilacosta I, et al. 2014. Symptomatic peripheral mycotic aneurysms due to infective endocarditis: a contemporary profile. Medicine Baltimore 93:42-52.

Gürel K, Gür S, Özkan U, Tekbaş G, et al. 2012. US-guided percutaneous thrombin injection of post catheterization pseudoaneurysms. Diagn Interv Radiol 18:319-25.

Leon LR, Psalms SB, et al. 2008. Infected Upper Extremity Aneurysms. A Review. Eur J Vasc En-dovasc Surg 35:320-331.

Mylonakis E, Calderwood SB. 2001. Infective endocarditis in adults. N Engl J Med 345:1318-1330.

Nishimura K, Hamasaki T, Yamamoto S, Kawai T, Sugiura K. 2015. Endovascular treatment of left subclavian artery pseudoaneurysm after clavicle fracture in an elderly adult with a 40-year history of Behçet's disease. Ann Vasc Dis 8:328-30.

Osler W. 1885. The Gulstonian Lectures on malignant endocarditis. $\mathrm{Br}$ Med J 1:467.

Patra P, Ricco J, Costargent A, Goueffic Y, et al. 2001. Association Universitaire de Recherche en Chirurgie (AURC). Infected aneurysms of neck and limb arteries: a retrospective multicenter study. Ann Vasc Surg 15:197-205.

Stengel A, Wolferth CC. 1923. Mycotic (bacterial) aneurysms of intravascular origin. Arch Intern Med (Chic) 31(4):527-554.

Stiru O, Geana CR, Pavel P, Croitoru M, Boros C, Iovu I, Iliescu, V. 2018. Descending thoracic aortic aneurysm rupture treated with thoracic endovascular aortic repair in a patient with peripheral artery disease. The Heart Surgery Forum 21(2): E112-E116.

Tsao JW, Marder SR, Goldstone J, et al. 2002. Presentation, diagnosis, and management of arterial mycotic pseudoaneurysms in injection drug users. Ann Vasc Surg 16:652-62. 